\title{
Relationship of Cerebral Palsy Subtypes and Activity
}

\section{Limitations}

Ozgun Kaya Kara ${ }^{1}$, Akmer Mutlu ${ }^{1}$, Mintaze Kerem Gunel ${ }^{1}$, Sevilay Karahan ${ }^{2}$, Ayşe Livanelioglu ${ }^{1}$ and Meral $\mathrm{Topcu}^{3}$

1. Department of Physiotherapy and Rehabilitation, Faculty of Health Sciences, Hacettepe University, 06100 Samanpazari, Ankara, Turkey

2. Faculty of Medicine, Department of Biostatistics, Hacettepe University, 06100 Samanpazari, Ankara, Turkey

3. Faculty of Medicine, Department of PediatricNeurology, Hacettepe University, 06100 Samanpazari, Ankara, Turkey

\begin{abstract}
Purpose: The aims of this study were to describe the relative proportions of cerebral palsy (CP) subtypes and associations between CP subtypes based on the Surveillance of Cerebral Palsy in Europe (SCPE) classification system; and to investigate the relationship between subtypes of $\mathrm{CP}$ and activity limitations as proposed in International Classification of Functional, Disability and Health (ICF). Method: The clinical types of the children were determined according to the definitions adopted for the SCPE. A total of 502 children with CP were identified. Activity limitations were studied with the Gross Motor Functional Classification System (GMFCS) for gross motor functionand the Manual Ability Classification System (MACS) for manual ability. The statistical analyses were performed with SPSS for Windows version 15.0. Results: GMFCS and MACS were associated with each other (r: 0.749, $\mathrm{p}<$ 0.001) regardless of the subtype. GMFCS and MACS were found to be associated in bilateral spastic, unilateral spastic, ataxic, dystonic and choreatetoid types (r: 0.86, r: 0.78, r: 0.70, r: 0.99, r: 0.82 respectively; $\mathrm{p}<0.05$ ). Conclusion: The study results stress the importance of merging information on CP subtypes, based on SCPE and functional motor evaluations. There is strong relationship between neurological subtype and activity limitations in children with $\mathrm{CP}$.
\end{abstract}

Key words: Cerebral Palsy, activity limitations, and classification

\section{Introduction}

A common language on Cerebral Palsy (CP) has been developed for the European registers by the SCPE (Surveillance of Cerebral Palsy in Europe) working group [1]. The first important achievement of SCPE was to establish a consensus of standards, definitions and classification systems of CP [1,2].

SCPE reached a consensus on the following definition that is a synopsis of the key elements of current CP definitions: $\mathrm{CP}$ is a group of permanent and non-progressive disorders of movement and posture and of motor function, caused by a non-progressive interference, lesion, or abnormality of the immature brain [3]. The classification of the

\footnotetext{
Corresponding author: Ozgun Kaya Kara, PT, MSc, research field: pediatric rehabilitation. E-mail: ozgun_kaya@yahoo.com.
}

heterogeneous group of children with CP has recently become more important [4].

Classifications have been made based on the anatomical site of the brain impairment; the clinical symptoms and signs, e.g., spastic conditions, dyskinesias or ataxias; the topographical involvement of the extremities, e.g., hemiplegia, diplegia, quadriplegia; the degree of muscle tone, e.g., isotonic, hypotonic or hypertonic; and the timing of the presumed insult, e.g., prenatal, perinatal or postnatal [5]. To these problems of definition and classification are added the variation over time of symptoms and signs in a child and the variation between the assessing clinicians and those interpreting these symptoms and signs [4].

A decision tree to enable a case to be allocated to a given type of CP has been formulated by SCPE [3]. In 
this SCPE decision tree, spastic $\mathrm{CP}$ is divided only into bilateral (BSCP) and unilateral (USCP) (hemiplegia) cases. The distinction between diplegia and tetraplegia has proven to be very difficult to define [6]. The Swedish Classification (SC) therefore does not separate spastic tetraplegia and diplegia and children are instead described according to the functional level in the lower and upper extremities, cognitive development, visual function, hearing, epilepsy, etc. [7]. Dyskinetic CP is divided into a tonus-changing (dystonic) and a choreo-athetoid type. According to the SCPE classification, dystonic $\mathrm{CP}$ is characterized by hypokinesia as well as a tendency to increased muscle tone, while hyperkinesia and a tendency to decreased muscle tone are typical of the choreo-athetoid subtype. Both subtype classifications (SCPE and SC) are based on the dominant neurological sign, although a mixed (SC) or non-classifiable (SCPE) category is available for exceptional cases. The development of SCPE has had a positive influence in standardizing and recording the examination of children with $\mathrm{CP}$ and in classifying the subtypes [4]. A consequence has been the development of the decision tree to improve the classification of $\mathrm{CP}$ subtypes [3].

The SCPE classification was developed to improve harmonization of data for monitoring the rates of $\mathrm{CP}$ and to provide a framework for research and service planning. The validity and reliability of the SCPE guidelines for the classification of $\mathrm{CP}$ was demonstrated by Gainsborough et al. [8].

The traditional clinical terminology used to describe CP subtypes has been confusing [9] and the standard form for describing children with a central motor deficit is an attempt to avoid these terms [10]. Gorter et al. pointed out that the elegant work of SCPE leaves open to judgment how to distinguish unilateral from bilateral $\mathrm{CP}$ with precision as well as how to distinguish diplegia from quadriplegia [11]. In a previous study, Kerem-Gunel and Mutlu investigated the disability and its relation with functional independence and suggested that the SCPE classification for CP subtypes is simple, practical and clear [12].

Children with $\mathrm{CP}$ comprise a very heterogeneous group. The SCPE therefore recommends describing functional severity in the legs and arms according to standardized scores such as the Gross Motor Function Classification System (GMFCS)[13] and the Bimanual Fine Motor Function System (BFMF) [14] and the Manual Ability Classification System (MACS) [15]. The classification of CP proposed by SCPE, supplemented by a description of gross and fine motor function, has been a useful tool for recording and describing the panorama of $\mathrm{CP}$, but the classification of children with mixed forms remains a challenge [16, 17].

The GMFCS was developed to describe gross motor function in children with $\mathrm{CP}$ and focuses on self-initiated movements, in particular sitting and walking [13]. The GMFCS has been internationally accepted and is widely used. The MACS classifies how well children aged 4-18 years with CP use their hands when handling objects in daily activities. It is designed to reflect the child's typical manual performance, not the maximal capacity [15].

The World Health Organization (WHO) is encouraging application of International Classification of Functioning, Disability and Health (ICF); as a classification tool and also as a framework for social policy, research, education and clinical practice. The approach to the assessment and management of children and youth with $\mathrm{CP}$ may depend to a considerable extent on frameworks used to conceptualize diseases and disorders. According to ICF, impairments were described as problems in body structures or functions such as spasticity and activity limitations were described as difficulties in activities and participation restrictions as problems in manner of involvement in daily life situations [12, 14].

The aims of this study were to describe the relative proportions of cerebral palsy (CP) subtypes and 
associations between $\mathrm{CP}$ subtypes based on the Surveillance of Cerebral Palsy in Europe (SCPE) classification system; and to inform clinicians about the motor functional levels according to the SCPE subtypes, to investigate the relationship between subtypes of $\mathrm{CP}$ and activity limitations as proposed in International Classification of Functional, Disability and Health (ICF).

\section{Method}

\subsection{Participants}

The participants of the present study were all children with $\mathrm{CP}$ aged from 2 to 18 years at study entry. The sampling method was a consecutive sample of convenience in which the children with $\mathrm{CP}$ were selected from our unit. Our unit is an outpatient clinical and research center and a reference point-accepting children with $\mathrm{CP}$ or the many other pediatric neurological disorders (spina bifida, high risk infants, mental motor retardations, etc.) together with their families from the entire country. A total of 1165 cases with various pediatric neurological disorders were referred to and seen at our center for physical therapy and rehabilitation between January 2008 and May 2010.

The inclusion criterion for the study was a definite diagnosis of $\mathrm{CP}$ by a pediatric neurology department. A total of 502 of the 1165 cases had a CP diagnosis, of whom 214 were younger than four years old. The exclusion criteria for the study were the presence of other disorders and no definite diagnosis of CP. Cases excluded from the study included 134 high-risk infants, 167 children with mental motor retardation and 280 with no definite CP diagnosis (Fig. 1).

Informed consent was obtained from all parents. Ethical approval for the study was given by the Hacettepe University Medical Faculty Ethics Committee (Registration Number: HEK 09/60).

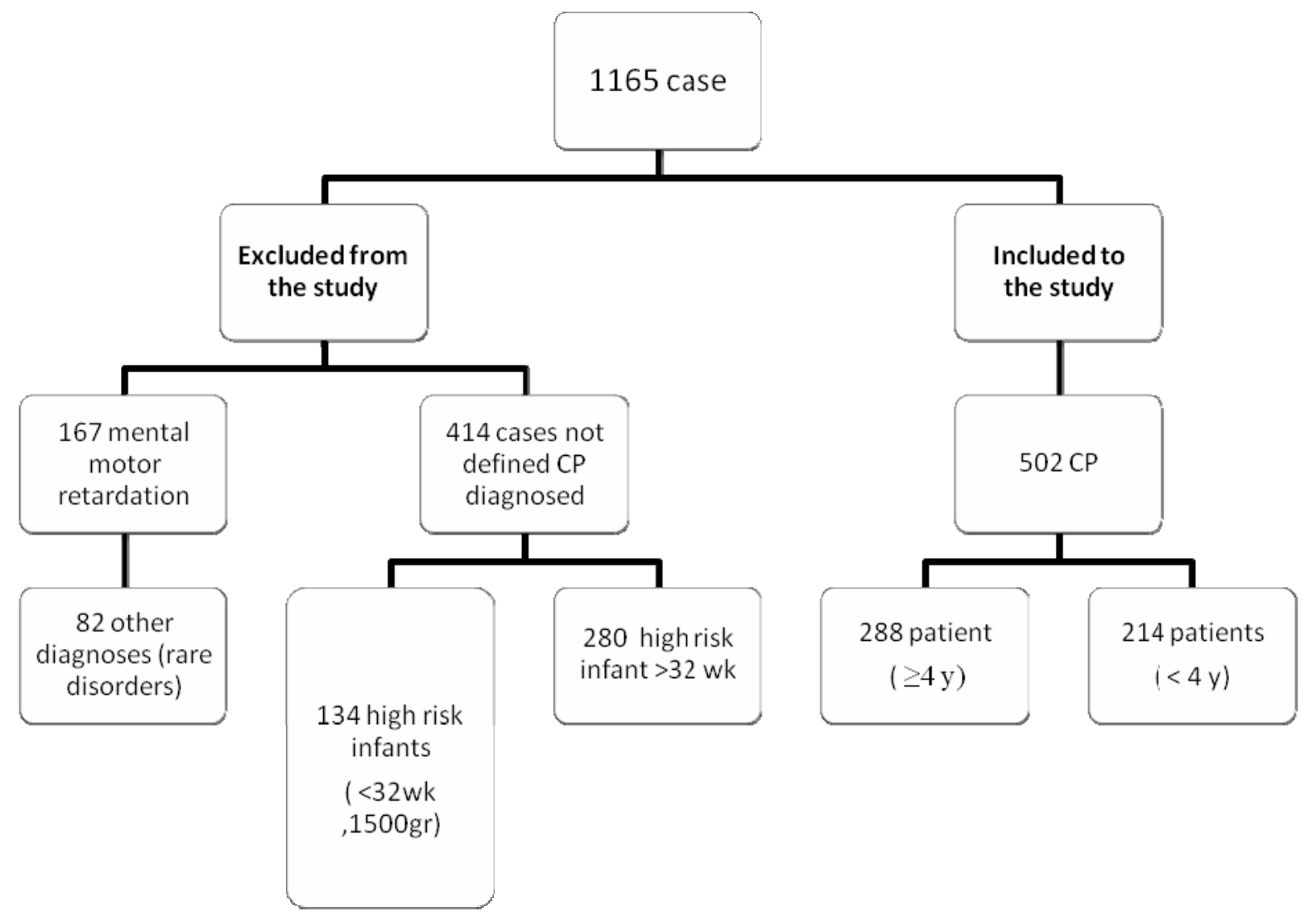

Fig. 1 Flow of participants through the study: Sample selection and recruitment. 


\subsection{Study Procedure}

All children attended the pediatric rehabilitation unit with their parent(s). One of two trained investigators (OKK/AM) classified the children during these visits according to the GMFCS and MACS level, type of motor impairment, and limb distribution. A standardized form was used to collect information for the children. The CP subtypes was determined by the responsible physician.

\section{(1) Case vignettes}

The vignettes included prenatal, natal, and postnatal medical records, and details of birth and early developmental history, birth asphyxia, kernicterus, neonatal convulsion, epilepsy, maternal disorder, vision disorder, speech and hearing disorder, learning disabilities. The following definitions of neuroimpairments were included: learning disability, defined as mild in children with IQ 50 to 70 and severe if the IQ was less than 50; epilepsy, defined as a diagnosis of epilepsy in the medical records; visual impairment, defined as acuity $\leq 0.3$ in the best eye with correction or presence of functional blindness; hearing impairment, defined as the need of a hearing aid or the presence of deafness [18].

(2) The clinical types of the children were determined according to definitions adopted for the European Classification of CP by the SCPE [3]. The patients were classified into the spastic, dyskinetic, ataxic, and unclassifiable subtypes according to the SCPE. To standardize the records as much as possible, the reporting clinicians were thoroughly informed of the SCPE guidelines on the definition and subclassification in a number of meetings and by receiving the R\&T Manual. It may thus be considered a strength of the study that the assessments of the children were performed by the clinicians who themselves examined the patients. In case a child showed a mixture of spastic, dyskinetic, and ataxic features, the predominant motor impairment subtype was chosen according to the SCPE framework.

\section{(3) Gross motor function}

The GMFCS is a common classification system and an evidence-based classification tool consisting of five levels ranging from Level I that includes children with minimal or no disability with respect to community mobility to Level $\mathrm{V}$ that includes children who are totally dependent on external assistance for mobility $[5,8,9]$. The expanded and revised GMFCS was developed in 2008 by adding a new age band of 12-18 years [9]. The GMFCS Expanded and Revised Version include five levels and five age bands: less than 2 years, 2 to 4 years, 4 to 6 years, 6 to 12 years and 12 to 18 years $[9,19]$. Gross motor function was further dichotomised by defining GMFCS levels I-III as "mild-moderate" and GMFCS levels IV-V as "severe" dysfunction.

\section{(4) Manual Ability}

The focus is on manual ability as defined in the International Classification of Functioning, Disability and Health [20] and the MACS level is influenced by environmental and personal factors. Good validity and reliability have been reported [21-23], and the MACS has already gained much international attention. It has been translated into 19 languages to date. Classification according to the MACS can be done at four years of age at the earliest. Consistent with the dichotomization of gross motor function, MACS levels I-III were defined as "mild-moderate" and MACS levels IV-V as "severe" dysfunction.

\section{Statistical Analysis}

The statistical analyses were performed with SPSS for Windows version 15.0 (SPSS Inc., Chicago, Illinois, USA). $\mathrm{P}$ values less than 0.05 were considered to be statistically significant. The relationship between the SCPE type and GMFCS-MACS was evaluated by the Chi square test. The relationship between subtypes of $\mathrm{CP}$ and activity limitations was analyzed using The Spearman correlation coefficient. The overall agreement between the GMFCS and MACS was analyzed using Kappa 
statistics. According to Altman, the Kappa value is to be interpreted as follows: $<0.20$ poor, $0.21-0.40$ fair, $0.41-0.60$ moderate, $0.61-0.80$ good, and $>0.80$ very good agreements [24].

\section{Results}

A total of 502 children with CP were identified. The mean age of the participants was 64.5 months (SD 42.3 mo., range of 24-216 mo.). The mean age of the mothers at delivery was 27.4 years (SD 5.9 years, range 15-48 years). The mean gestational age of the patients was $36 \mathrm{wk}$. (SD $4.7 \mathrm{wk}$., range 24-42 wk.). The mean birth weight of the cases was 2678 gr. (SD 981.3 gr., range 640-6500 gr.). The male-female distribution was 58.4\% (293) males and 41.6\% (209) females. The CP type distribution was spastic bilateral in $63 \%(320 / 502)$ and spastic unilateral in $28.1 \%$
(141/502). Detailed descriptive and comorbidity data of the children with CP are shown in Table 1.

According to GMFCS, 22.7\% (114/502) of the children were classified as Level I, 21.5\% (108/502) as Level II, $12.5 \%(63 / 502)$ as Level III, $22.3 \%$ $(112 / 502)$ as Level IV and 20.9\% (105/502) as Level V. According to MACS, $40.6 \%(117 / 288)$ of the children were classified as Level I, 16.3\% (47/288) as Level II, 14.9\% (43/288) as Level III, 7.6\% (22/288) and $20.9 \%(59 / 288)$ as Level V.

The association between the GMFCS levels and the SCPE classification is shown in Table 2. Levels I-II and III are combined for "mild-moderate" and Level IV and V for "severe" functional levels. According to the GMFCS, $41 \%(134320)$ of the bilateral spastic children were at levels I to III while 94\% (133/141) of

Table 1 Descriptive characteristics and comorbidity conditions of children with CP.

\begin{tabular}{|c|c|c|c|}
\hline & & $\mathrm{n}$ & $\%$ \\
\hline \multirow{5}{*}{ Classification of SCPE } & Spastic bilateral & 320 & 63.7 \\
\hline & Spastic unilateral & 141 & 28.1 \\
\hline & Ataxic & 11 & 2.2 \\
\hline & Dystonic & 15 & 3.0 \\
\hline & Choreoathetotid & 15 & 3.0 \\
\hline \multirow{2}{*}{ Gender } & Girls & 209 & 41.6 \\
\hline & Boys & 293 & 58.4 \\
\hline \multicolumn{2}{|c|}{ Maternal disorder } & 59 & 11.8 \\
\hline \multicolumn{2}{|c|}{ Medication } & 15 & 3.0 \\
\hline \multicolumn{2}{|c|}{ Smoking } & 62 & 12.4 \\
\hline \multicolumn{2}{|c|}{ RH incompatibility } & 45 & 9.0 \\
\hline \multirow[t]{2}{*}{ Delivery method } & Vaginal & 287 & 57.2 \\
\hline & Caesarean & 215 & 42,8 \\
\hline \multicolumn{2}{|c|}{ Birth asphyxia } & 200 & 39.8 \\
\hline \multicolumn{2}{|c|}{ Cyanosis } & 196 & 39.0 \\
\hline \multicolumn{2}{|c|}{ Crying } & 251 & 50.0 \\
\hline \multicolumn{2}{|c|}{ Neonatal convulsion, epilepsy } & 169 & 33.7 \\
\hline \multicolumn{2}{|c|}{ Kernicterus } & 153 & 30.5 \\
\hline \multicolumn{2}{|c|}{ Blood transfer } & 25 & 5,0 \\
\hline \multicolumn{2}{|c|}{ Drug utilization } & 195 & 38.8 \\
\hline \multicolumn{2}{|c|}{ Vision disorder } & 139 & 27.7 \\
\hline \multicolumn{2}{|c|}{ Speech disorder } & 196 & 39.0 \\
\hline \multicolumn{2}{|c|}{ Hearing disorder } & 43 & 8.6 \\
\hline \multicolumn{2}{|c|}{ Learning disability } & 182 & 36.3 \\
\hline
\end{tabular}


Table 2 Association between GMFCS and SCPE classifications.

\begin{tabular}{|c|c|c|c|c|c|c|}
\hline & & LEVEL 1 & LEVEL 2 & LEVEL 3 & LEVEL 4 & LEVEL 5 \\
\hline \multirow{6}{*}{ GMFCS } & Spastic bilateral & $37(11.6 \%)$ & $46(14.4 \%)$ & $51(15.9 \%)$ & $101(31.6 \%)$ & $85(26.6 \%)$ \\
\hline & Spastic unilateral & $72(51.1 \%)$ & $53(37.6 \%)$ & $8(5.7 \%)$ & $4(2.8 \%)$ & $4(2.8 \%)$ \\
\hline & Ataxic & $4(36.4 \%)$ & $5(45.5 \%)$ & $2(18.2 \%)$ & $0(0.0 \%)$ & $0(0.0 \%)$ \\
\hline & Dystonic & $0(0.0 \%)$ & $3(20.0 \%)$ & $1(6.7 \%)$ & $2(13.3 \%)$ & $9(60.0 \%)$ \\
\hline & Choreoathetotid & $1(6.7 \%)$ & $1(6.7 \%)$ & $1(6.7 \%)$ & $5(33.3 \%)$ & $7(46.7 \%)$ \\
\hline & Test result & \multicolumn{5}{|c|}{$\chi^{2}=215,804, p<0.001$} \\
\hline \multirow{6}{*}{ MACS } & Spastic bilateral & $78(44.6 \%)$ & $18(10.3 \%)$ & $16(9.1 \%)$ & $18(10.3 \%)$ & $45(25.7 \%)$ \\
\hline & Spastic unilateral & $34(41.5 \%)$ & $22(26.8 \%)$ & $22(26.8 \%)$ & $2(2.4 \%)$ & $2(2.4 \%)$ \\
\hline & Ataxic & $5(50.0 \%)$ & $3(30.0 \%)$ & $2(20.0 \%)$ & $0(0.0 \%)$ & $0(0.0 \%)$ \\
\hline & Dystonic & $0(0.0 \%)$ & $3(25.0 \%)$ & $2(16.7 \%)$ & $1(8.3 \%)$ & $6(50.0 \%)$ \\
\hline & Choreoathetotid & $0(0.0 \%)$ & $1(11.1 \%)$ & $1(11.1 \%)$ & $1(11.1 \%)$ & $6(66.7 \%)$ \\
\hline & Test result & \multicolumn{5}{|c|}{$\chi^{2}=83,618, p<0.001$} \\
\hline
\end{tabular}

Table 3 Agreement between GMFCS and MACS levels according to SCPE subtypes.

\begin{tabular}{|c|c|c|c|c|c|}
\hline & $\begin{array}{c}\mathrm{r} \text { (Spearman Correlation } \\
\text { Coefficient) }\end{array}$ & $\begin{array}{c}\text { Overall } \\
\text { Agreement }\end{array}$ & Kappa & $95 \%$ CI for Kappa & $\mathrm{p}$ \\
\hline Spastic bilateral & 0.86 & $50 \%$ & 0.39 & $0.33-0.46$ & $<0.001$ \\
\hline Spastic unilateral & 0.78 & $51 \%$ & 0.26 & $0.16-0.34$ & $<0.001$ \\
\hline Ataxic & 0.70 & $60 \%$ & 0.39 & $0-0.83$ & 0.066 \\
\hline Dystonic & 0.99 & $92 \%$ & 0.87 & $0.72-1.00$ & $<0.001$ \\
\hline Choreoathetotid & 0.82 & $78 \%$ & 0.65 & $0.56-0.84$ & 0.001 \\
\hline Total cases & 0.75 & $53 \%$ & 0.41 & $0.37-0.45$ & $<0.001$ \\
\hline
\end{tabular}

the unilateral spastic children were at levels I to III. Of the children with dystonic CP, $73.3 \%$ (11/15) were at levels IV or V. Of the children with choreo-athetoid CP, $80 \%(12 / 15)$ were at levels IV or V. The relationship between SCPE and GMFCS levels was found to be significant. Most BSCP (bilateral spastic cerebral palsy), dystonic and choreo-athetoid children were in the "severe" group (Level IV-V) while most USCP (unilateral spastic cerebral palsy) children were in the "mild-moderate" group (Level I-II-III).

According to the MACS, 64\% (112/20) of the bilateral spastic children were at levels I to III while 95.1\% (133/141) of the unilateral spastic children were at levels I to III. Of the children with dystonic CP, $58.3 \%$ (7/15) were at levels IV or V. Of the children with dystonic CP, 78.8\% (7/15) were at levels IV or V. The relationship between SCPE and MACS levels was found to be statistically significant. Most BSCP, dystonic and choreo-athetoid children were in the "severe" group (Level IV-V) while most USCP children were in the "mild-moderate" group (Level I-II-III).

Agreement between the GMFCS and MACS levels for SCPE subtypes is shown in Table 3. According to these subtypes, GMFCS and MACS had a positive relationship in bilateral spastic, unilateral spastic, dystonic and choreo-athetoid types (r: 0.86, r: 0.78, r: 0.99 , r: 0.82 respectively; $\mathrm{p}<0.001$ ).

\section{Discussion}

The SCPE network has recently agreed upon a definition of $\mathrm{CP}$, and has suggested a revised classification of the subtypes that may be less dependent on individual judgment [3]. In the present study showed to value of SCPE classification systems in referral-based consecutive sample of convenience.

Many investigators have studied the relationship between $\mathrm{CP}$ subtypes and associated impairments 
(vision, hearing, speech disorder, mental retardation etc.) $[12,16,25]$. Beckung et al. found that the type of impairment was associated with the type of CP [18]. Mental retardation and epilepsy were most frequent and seen in $40 \%$ and $35 \%$, respectively in the Beckung et al study. The learning disability rate was $36.3 \%$, and the epilepsy rate $33.7 \%$ in our study. These results are compatible with the Hagberg et al., Nordmark et al, and Mutlu et al studies [12, 26-28]. In the Andersen study, the rate for mental retardation was $31 \%$, speech disorder $38 \%$, vision disorder $5 \%$, hearing disorder $4 \%$, and epilepsy $28 \%$ [16]. Our results are still higher than those reported by Andersen et al at $36 \%, 39 \%, 27 \%, 8 \%$, and $33 \%$ respectively. This might be due to the larger sample size of our study compared with the Andersen et al study.

In the present study, the highest number of participants was in the BSCP type and the lowest number in the ataxic type. The Krageloh-Mann study indicated the subtypes according to the SCPE classification system. They found $88 \%$ had spastic CP [58\% bilateral, 30\% unilateral), $7 \%$ dyskinetic $\mathrm{CP}$, and $4 \%$ ataxic $\mathrm{CP}$ while $1 \%$ were not classifiable [1]. In the Andersen study, 33\% of the 294 children had spastic unilateral CP, 49\% spastic bilateral CP, 6\% dyskinetic $\mathrm{CP}$ and 5\% ataxic CP [16]. In the Carnahan study, $79 \%$ of the total sample of 359 children had spastic CP (42.7\% unilateral and $57.3 \%$ bilateral), $14 \%$ dyskinetic $\mathrm{CP}$ and $2 \%$ ataxic $\mathrm{CP}$ according to the SCPE system [9]. Our results were essentially similar to other studies. The common point of all studies was that the spastic type of $\mathrm{CP}$ was the most frequent type.

The GMFCS classification alone is not a valid indicator of anything other than motor function abilities, even if the frequency and severity of associated impairments increase in line with higher GMFCS disability levels in group comparisons [14, 25, 27, 28]. Accordingly, the total impairment load of children with $\mathrm{CP}$ correlates with the SCPE sub classification when combined with the GMFCS levels [27]. Andersen et al. demonstrated that the classification of $\mathrm{CP}$ as proposed by SCPE, supplemented by a description of gross and fine motor function, was a useful tool for recording and describing the panorama of CP in Norway, but they stated that the classification of children with mixed forms remains a challenge [16]. In the present study, the distribution of the 502 children with $\mathrm{CP}$ was proportional between the GMFCS levels. This may be result of USCP representing mostly the milder affected spastic CP group while BSCP represents the severely dysfunctional spastic CP group. Thus, all the levels have similar percentages.

In the present study, there were 222 (44.22\%) children in GMFCS I+II and 164 (56.9\%) who were independent concerning manual ability in age-relevant daily activities (MACS I+II) in this study, In the Carnahan study, there were 220 children (61\%) who could walk independently (GMFCS I+II) and 231 children $(64 \%)$ who were independent concerning manual ability in age-relevant daily activities (MACS I+II) [7]. These results differ because our aim was to investigate the relationship between $\mathrm{CP}$ subtypes and GMFCS-MACS. Therefore the GMFCS level was indicated in 502 children while the MACS level was identified in 288 children.

Most USCP cases had "mild-moderate" gross motor function and manual ability impairment whereas BSCP cases were mostly in the "severe" group but with some cases in the "mild-moderate" levels. However, patients with the dyskinetic type had severe functional limitations (GMFCS and MACS IV and V). Children with ataxia constituted a small group in the present study $(2.2 \%)$ andmost had mild-moderate motor function (GMFCS-MACS I-II-III). In the Westbom study, 12 out of the 19 children with choreo-athetosis as the dominant neurological sign but none of the children with dystonia had mild gross motor dysfunction (GMFCS III) [29]. In the Carnahan study, 34 (67\%) of the children with dyskinetic CP (n $=51)$ had very limited self-mobility (GMFCS IV-V) as well as limited manual ability (MACS IV-V) [7]. 
These results were consistent with the literature and showed that the dyskinetic type of $\mathrm{CP}$ is the more severe type.

In this study, severely impaired gross motor function (GMFCS: IV-V) was present in $8(5.7 \%)$ unilateral CP cases compared with 186 (58.1\%) children with bilateral $(\mathrm{p}<0.01)$ and $23(76.6 \%)$ of children with dyskinetic CP $(\mathrm{p}<0.01)$. Andersen et al found severely impaired gross motor function (GMFCS: IV-V) in $1(1 \%)$ child with unilateral CP compared with $53(38 \%)$ children with bilateral $(\mathrm{p}<$ $0.01)$ and $16(89 \%)$ children with dyskinetic CP $(\mathrm{p}<$ 0.01 vs. unilateral) [16].

In the Carnahan study, the two different non-parametric tests yielded consistent results regarding the association between GMFCS and MACS in the CP subtypes [7]. The Shevell et al study found a high correlation between gross motor function and CP subtype [30]. In the present study, there was a significant relationship between the SCPE and MACS, SCPE and GMFCS levels.

The different distribution of GMFCS and MACS with the SCPE system is caused by the BSCP group containing both di- and tetraplegic CP (SC) with diplegic $\mathrm{CP}$ having better manual ability than tetraplegic cases. Carnahan et al found various associations in the $\mathrm{CP}$ subtypes. They identified that manual ability was more limited than gross motor function in hemiplegic $\mathrm{CP}$ while the opposite was found in children with diplegic CP [7].

The term bilateral $\mathrm{CP}$, suggested by the SCPE group, combines spastic diplegia and tetraplegia, which further stresses the need for additional functional grading in order to correctly evaluate each child. A structured model for such a functional grading was also suggested by the SCPE group and may be very useful when describing populations of children with CP [7].

One of the limitations of our study was not being able to compare the ataxic and dyskinetic types of $\mathrm{CP}$, as we had only small numbers of cases in these groups, with spastic CP. The other limitation of our study is that the cognitive level of the children might have affected the classification levels.

In conclusion, the SCPE classification system is a useful and practical system in the clinic and also eliminated the difficulty in assigning children to di-or tetraparetic CP.The next step of this study should be larger groups in subtypes of $\mathrm{CP}$ are required.

\section{References}

[1] I. Krageloh-Mann and C. Cans, Cerebral palsy update, Brain Dev. 31 (2009) 537-544.

[2] C. Christine, H. Dolk, M. J. Platt, A. Colver, A. Prasauskiene and I. Krageloh-Mann, SCPE collaborative group: Recommendations from the SCPE collaborative group for defining and classifying cerebral palsy, Dev Med Child Neurol 109 (2007) 35-38.

[3] SCPE working group, Surveillance of cerebral palsy in Europe: A collaboration of cerebral palsy surveys and registers, Surveillance of Cerebral Palsy in Europe (SCPE). Dev Med Child Neurol 42 (2000) 816-824.

[4] P. O. Pharoah, Dyskinetic cerebral palsy in Europe: Trends in prevalence and severity, on behalf of the SCPE Collaboration, ArchDis Child 94 (2009) 917-918.

[5] M. A. Perlstein, Infantile cerebralpalsy; classification and clinical correlations, JAMA 149 (1952) 30-34.

[6] A. F. Colver and T. Sethumadhavan, The termdiplegian should be abandoned, ArchDis Child 88 (2003) 286-290.

[7] K. D. Carnahan, M. Arner and G. Hagglund, Association between gross motor function (GMFCS) and manual ability (MACS) in children with cerebral palsy: A population-based study of 359 children, BMC Musculoskelet Disord. 8 (2007) 50.

[8] M. Gainsborough, G. Surman, G. Maestri, A. Colver and C. Cans, Validity and reliability of the guidelines of the surveillance of cerebral palsy in Europe for the classification of cerebral palsy, Dev Med Child Neurol. 50 (2008) 828-831.

[9] T. T. S., Ingram A historical review of the definition and classification of the cerebralpalsies, in: Fiona Stanley and Eva Alberman (Eds.), The Epidemiology of the Cerebral Palsies, Oxford: Blackwell Scientific (1984) 1-11.

[10] P. Evans, A. Johnson, L. Mutch and E. Alberman, A standart form for recording clinical findings in children with a motor deficit of central origin, Dev Med Child Neurol. 31 (1989) 119-127.

[11] J. W. Gorter, P. L. Rosenbaum, S. E. Hanna, R. J. Palisano, D. J. Bartlett, D. J. Russell, S. D. Walter, P. Raina, B. E. Galuppi and E. Wood, Limb distribution 
motor impairment, and functional classification of cerebral palsy, Dev Med Child Neurol. 6 (2004) 461-467.

[12] A. Mutlu, P. P. Akmese, M. K. Gunel, S. Karahan and A. Livanelioglu, Theimportance of motor functional levels from the activity limitation perspective of ICF in children with cerebral palsy, Int J RehabilRes 33 (4) 2010 319-324.

[13] R. Palisano, P. Rosenbaum, S. Walter, D. Russell, E. Wood and B. Galuppi, Development and reliability of a system to classify gross motor function in children with cerebral palsy, Dev Med Child Neurol 39 (1997) 214-223.

[14] E. Beckung and G. Hagberg, Correlation between ICIDH handicap code and Gross Motor Function Classification System in children with cerebral palsy, Dev Med Child Neurol 42 (2000) 669-673.

[15] A. C. Eliasson, L. Krumlinde-Sundholm, B. Rosblad, E. Beckung, M. Arner and A. M. Ohrvall et al., The Manual Ability Classification System (MACS) for children with cerebral palsy: scale development and evidence of validity and reliability, Dev Med Child Neurol 48 (2006) 549-554.

[16] G. L Andersen, L. M. Irgens, I. Haagaas, J. S. Skranes, A. E. Meberg and T. Vik, Cerebral palsy in Norway: prevalence, subtypes and severity, Eur J Paediatr Neurol. 12 (1) (2008) 4-13.

[17] P. Rosenbaum, J. W. Gorter, R. Palisano and C. Morris The relationship of cerebral palsy subtype and functional motor impairment: A population-based study, Dev Med Child Neurol. 52 (7) (2010) 682-683.

[18] E. Beckung and G. Hagberg, Neuro impairments, activity limitations, and participation restrictions in children with cerebral palsy, Dev Med Child Neurol 44 (2002) 309-316.

[19] International classification of functioning, disability and health, Geneva: World Health Organisation, 2001.

[20] A. Mutlu, O. K. Kara, M. K. Gunel, S. Karahan and A. Livanelioglu, Agreement between parents and clinicians for the motor functional classification systems of children with cerebral palsy, Disabil Rehabil. 33 (11) (2011) 927-932.
[21] C. Morris, J. Kurinczuk, R. Fitzpatrick and P. Rosenbaum, Reliability of the Manual Ability Classification System for children with cerebral palsy, Dev Med Child Neurol 48 (2006) 950-953.

[22] P. Akpinar, C. G. Tezel, A. C. Eliasson and A. Icagasioglu, Reliability and cross-cultural validation of the Turkish version of Manual Ability Classification System (MACS) for children with cerebral palsy, Disabil Rehabil. 32 (23) (2010) 1910-1916.

[23] J. Van Meeteren, C. Nieuwenhuijsen, A. de Grund, H. J. Stam and M. E. Roebroeck, Using the manual ability classification system in young adults with cerebral palsy and normal intelligence, Disabil. Rehabil. 32 (23) (2010) 1885-1893.

[24] D. G. Altman, Practical Statistics Formedical Research (9th ed.), Chapman\&Hall, London; 1997, pp. 230, 404-415.

[25] K. Himmelmann, E. Beckung, G. Hagberg and P. Uvebrant, Gross and fine motor function and accompanying impairments in cerebral palsy, Dev Med Child Neurol 48 (2006) 417-423.

[26] B. Hagberg, G. Sanner and M. Steen, The dys equilibrium syndrome in cerebral palsy: Clinical aspects and treatment, Acta Paediatr Scand Suppl 226 (1972) $1-63$.

[27] E. Nordmark, G. Hagglund and J. Lagergren, Cerebral palsy in Southern Sweden - Prevalence and clinical features (Part I), Acta Paediatr 90 (2001) 1271-1276.

[28] E. Nordmark, G. Hagglund and J. Lagergren, Cerebral palsy in Southern Sweden - Gross motor function and disabilities (Part II), Acta Paediatr 90 (2001) 1277-1282.

[29] L. Westbom, G. Hagglund and E. Nordmark, Cerebral palsy in a total population of 4-11 year olds in Southern Sweden: Prevalence and distribution according to different CP classification systems, BMC Pediatr. 5 (2007) $7-41$.

[30] M. I. Shevell, L. Dagenais and N. Hall, The relationship of cerebral palsy subtype and functional motor impairment: A population- based study, Dev Med Child Neurol 51 (2009) 872-877. 